\title{
Endothelial Cell Behavior Is Determined by Receptor Clustering Induced by Thrombospondin-1
}

\author{
Verônica Morandi ${ }^{1}$, Jim Petrik ${ }^{2 \star}$ and Jack Lawler ${ }^{3 *}$ \\ ${ }^{1}$ Rio de Janeiro State University (UERJ), Rio de Janeiro, Brazil, ${ }^{2}$ University of Guelph, Guelph, ON, Canada, ${ }^{3}$ Beth Israel \\ Deaconess Medical Center, Harvard Medical School, Boston, MA, United States
}

The thrombospondins (TSPS) are a family of multimeric extracellular matrix proteins that dynamically regulate cellular behavior and response to stimuli. In so doing, the TSPs directly and indirectly affect biological processes such as embryonic development, wound healing, immune response, angiogenesis, and cancer progression. Many of the direct effects of Thrombospondin 1 (TSP-1) result from the engagement of a wide range of cell surface receptors including syndecans, low density lipoprotein receptorrelated protein 1 (LRP1), CD36, integrins, and CD47. Different or even opposing outcomes of TSP-1 actions in certain pathologic contexts may occur, depending on the structural/functional domain involved. To expedite response to external stimuli, these

Edited by:

Bin Ren,

University of Alabama at Birmingham,

United States

Reviewed by:

David D. Roberts,

National Institutes of Health (NIH),

United States

Jun Feng,

Alpert Medical School, Brown

University, United States

${ }^{*}$ Correspondence:

Jack Lawler

jlawler@bidmc.harvard.edu

Jim Petrik

jpetrik@uoguelph.ca

Specialty section:

This article was submitted to

Signaling,

a section of the journa

Frontiers in Cell and Developmental

Biology

Received: 05 February 2021

Accepted: 10 March 2021

Published: 29 March 2021

Citation:

Morandi V, Petrik J and Lawler J (2021) Endothelial Cell Behavior Is Determined by Receptor Clustering Induced by Thrombospondin-1. Front. Cell Dev. Biol. 9:664696. doi: 10.3389/fcell.2021.664696 receptors, along with vascular endothelial growth factor receptor 2 (VEGFR2) and Src family kinases, are present in specific membrane microdomains, such as lipid rafts or tetraspanin-enriched microdomains. The molecular organization of these membrane microdomains and their constituents is modulated by TSP-1. In this review, we will describe how the presence of TSP-1 at the plasma membrane affects endothelial cell signal transduction and angiogenesis.

Keywords: angiogenesis, CD36, syndecan, thrombospondin, CD47, integrin, endothelial cell

\section{INTRODUCTION}

Like most biological processes, formation of the vasculature is temporally and spatially regulated by a balance of the signals that are elicited by stimulators and inhibitors. The temporal changes in the levels of these factors in the microenvironment determines endothelial cell behavior. In normal adult tissue, the endothelium is in a quiescent state, but it can respond rapidly to form new capillaries through a process termed angiogenesis. To facilitate a rapid response, the constituents of the pro- and anti-angiogenic pathways are co-localized to specific regions of the plasma membrane, such as lipid rafts and tetraspanin-enriched microdomains (Garcia-Parajo et al., 2014). The recruitment of specific proteins and lipids to these clusters is a critical determinant of their function. This spatial compartmentalization of the pathway components facilitates the efficient regulation of signal transduction that is essential for correct physiological response.

Thrombospondin 1 (TSP-1) is a founding member of the matricellular family of proteins (Adams and Lawler, 2011). These proteins are expressed primarily at the cell surface where they participate in the dynamic changes that cells undergo in response to extracellular stimuli. TSP-1 is involved in cell-to-cell junctions in synapses and immune cell interactions (Soto-Pantoja et al., 2015; Risher et al., 2018). As an example of the simplest form of cell-to-cell interaction, TSP-1 forms a molecular bridge between integrins on apoptotic neutrophils and CD36 on macrophages (Ren and Savill, 1995). TSP-1 is also highly expressed in supramolecular attack particles that are 
made by cytotoxic T lymphocytes (Balint et al., 2020). A molecular layer of TSP-1 on the attack particles presumably functions to engage the target cell (Balint et al., 2020). TSP-1 also participates in the lateral association of membrane proteins within a single cell, such as endothelial cells where it affects the association of proteins involved in angiogenesis pathways. Taken together, the data indicate that TSP-1 brings membrane proteins together, either on the same cell or adjacent cells, to regulate cellular behavior.

Thrombospondin 1 is a large multimeric extracellular matrix protein that is expressed at sites of normal tissue development, remodeling, and repair (Adams and Lawler, 2011). In cutaneous wounds, TSP-1 is deposited by platelets to accelerate wound closure (Agah et al., 2002). Its coordinated expression with vascular endothelial growth factor (VEGF) is important for normal ovarian follicular development (Michaely et al., 2004). As a physiological activator of latent TGF $\beta$, TSP-1 is also involved in fibrotic and immune response (Murphy-Ullrich and Suto, 2018). Additionally, TSP-1 expression is altered during pathological conditions such as myocardial infarction and cancer (Frangogiannis et al., 2005; Stenina-Adognravi et al., 2018).

Thrombospondin 1 antagonizes angiogenesis through multiple mechanisms, several of which suppress the bioavailability of VEGF. TSP-1 inhibits MMP9 activity and suppresses the release of VEGF from the extracellular matrix (Rodriguez-Manzaneque et al., 2001). TSP-1 also binds VEGF and supports its clearance through an lipoprotein receptorrelated protein (LRP)-dependent mechanism (Greenaway et al., 2007). A significant portion of the direct anti-angiogenic activity of TSP-1 maps to the three, thrombospondin type 1 repeats, designated 3TSR (Russell et al., 2015). These repeats, which were first identified in TSP-1, contain binding sites for TGF $\beta$ and CD36 (Tan et al., 2002). As described below, the binding of TSP-1 to CD36 activates apoptosis pathways in endothelial cells.

The ability of TSP-1 to strongly bind heparin has long been identified and used as a strategic tool for purifying the protein from human platelets (Lawler et al., 1978; Sipes et al., 2018). Physiologically, this affinity for heparin may reflect the capacity of TSP-1 to bind to heparan sulfate (HS) and to heparan sulfate proteoglycans (HSPG; Sun et al., 1989). Binding to HS/HSPG has direct consequences for the role played by TSP-1 in endothelial cell adhesion, proliferation, motility, differentiation, and in the modulation of activity of angiogenic growth factors (Vischer et al., 1988; Vogel et al., 1993; Nunes et al., 2008).

The globular pentraxin-like heparin-binding N-terminal domain (HBD) of TSP-1 bears the main high-affinity sites for binding to heparin/HS (Clezardin et al., 1997). A prototypical BBXB consensus heparin/HS-binding motif (where $B$ represents basic amino acids) in the $\mathrm{HBD}$ is represented by the amino acid sequence MKKTRG (residues 79-84), while the sequences ARKGSGRR (residues 22-29) and TRDLASIARLRIAKGVNDNF (residues 170-189) lack this BBXB configuration. However, both are enriched in positively charged residues, essential for the electrostatic interactions playing a major role in the binding of heparin/HS to proteins (Munoz and Linhardt, 2004). Heparin-binding sites have also been identified in the three type-I repeats or properdin-like domains (Guo et al., 1992; Yu et al., 2000) but, while studies have confirmed the essential requirement for the HBD in the interaction of TSP-1 with heparin/HS chains, it remains unclear whether these secondary heparin binding sites are indeed functional in the intact molecule (Yu et al., 2000). So far, TSP-1 interactions with perlecan (Vischer et al., 1997; Feitsma et al., 2000), decorin (Winnemoller et al., 1992; Merle et al., 1997), and some members of the syndecan (SDC) family of HSPG (see below) have already been described.

In this review, we will focus on the well-characterized interactions of TSP-1 with SDCs, lipoprotein receptor-related protein 1 (LRP1), CD36, integrins, and CD47 in the context of endothelial cell phenotype and angiogenesis (Figure 1). We will discuss how the downstream signals elicited by engagement of these receptors are integrated to determine endothelial cell behavior.

\section{SYNDECANS AS RECEPTORS FOR TSP-1}

Mammalian SDCs comprise four cell surface members bearing type-I (single-pass) core proteins, with highly conserved short cytoplasmic domains, a transmembrane domain and a varying length extracellular domain, covalently substituted with HS chains (chondroitin or dermatan-sulfate chains may also be present occasionally, to a lesser extent) (Couchman, 2003). SDCs act as receptors or coreceptors for extracellular matrix, growth factors, chemokines, interleukins, and morphogens (Chung et al., 2016) and can engage laterally with other classes of receptors, such as integrins and tyrosine kinase receptors for growth factors (FGFR1, EGFR, among others) (Couchman, 2003), thus contributing to the triggering and modulation of pivotal signaling including $\mathrm{PKC} \alpha$, Akt, and Wnt pathways (Gondelaud and Ricard-Blum, 2019). Indeed, there is growing evidence that SDCs are key parts of processes that involve tissue remodeling, such as development, cancer, inflammation, and tissue repair (Chung et al., 2016). TSP-1 bound to a HSPG recognized by a monoclonal antibody later shown to react specifically with the SDC1 isoform, in mammary epithelial cells (Sun et al., 1989). Both molecules were also found colocalized during lung development (Corless et al., 1992).

Although all four isoforms of SDC have been to some extent implicated in the modulation of endothelial angiogenic differentiation (De Rossi and Whiteford, 2014), a major role for syndecan-4 (SDC-4) seems to prevail over other SDC isoforms, in the context of angiogenesis associated with inflammatory and mechanical stimuli, as well as with injuries occurring in the cardiovascular system (Matsui et al., 2011; Vuong et al., 2015; Russo et al., 2020). SDC-4-null mice exhibit delayed dermal wound repair and defective angiogenesis (Echtermeyer et al., 2001).

Among SDCs, SDC-4 isoform has a unique role in the formation of fibronectin (FN)-induced focal adhesions, in cooperation with $\beta 1$-integrin receptors (Woods and Couchman, 1994). As other matricellular proteins found in the ECM, TSP1 destabilizes focal adhesions (Murphy-Ullrich and Sage, 2014), 


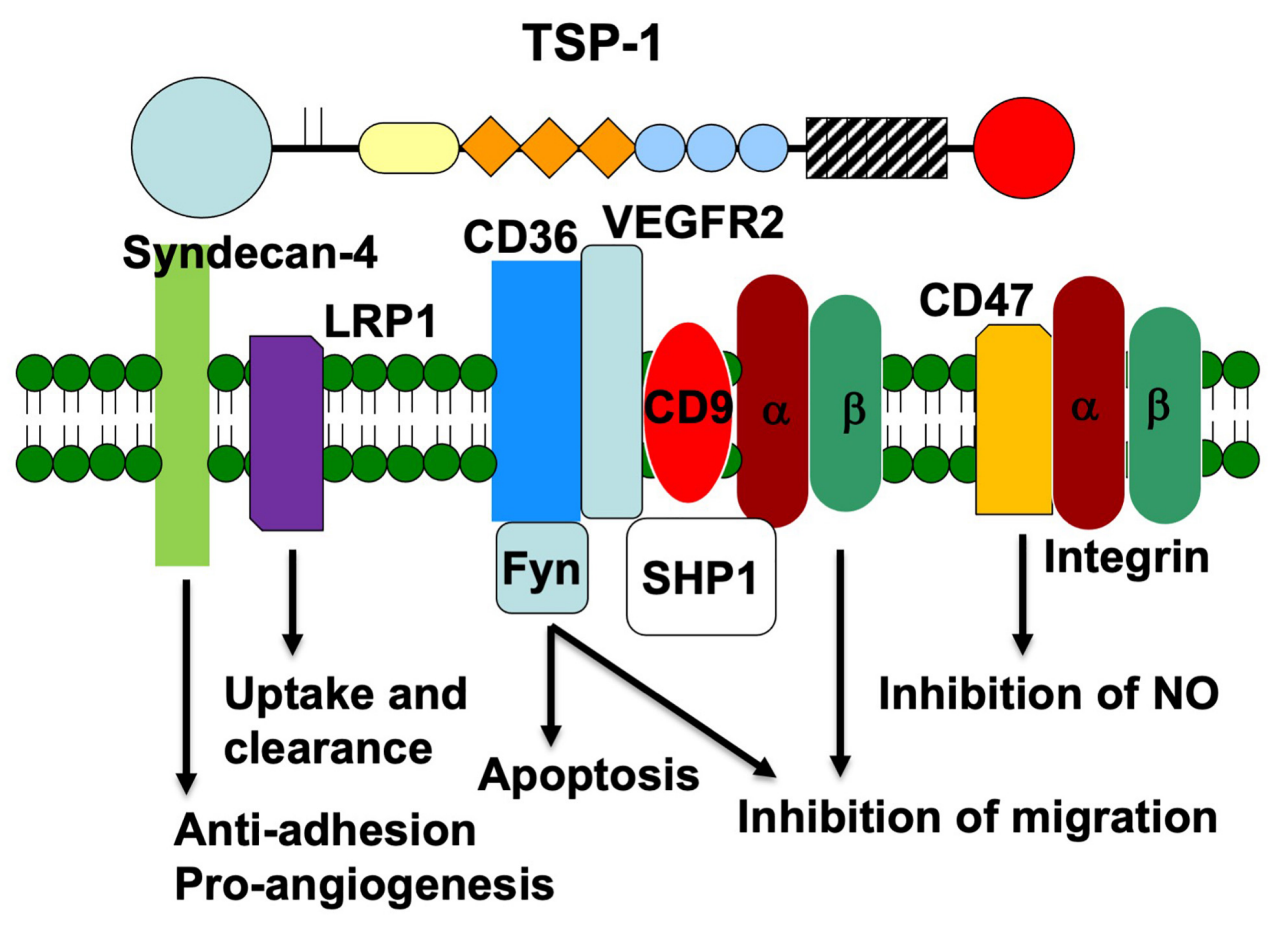

FIGURE 1 | Schematic representation of the endothelial cell receptors that interact with the various domains of TSP-1. The receptors are placed under the domain with which they interact. The signaling proteins that associate with each receptor and the signal that is transduced are also indicated.

and this activity was specifically located in the HBD domain of TSP-1, in endothelial models (Murphy-Ullrich et al., 1993; Vogel et al., 1993). Interestingly, pro-angiogenic activities of TSP1 have also been attributed to HBD (Chandrasekaran et al., 2000; Taraboletti et al., 2000; Ferrari do Outeiro-Bernstein et al., 2002).

A $18 \mathrm{kDa} \mathrm{HBD}$ comprising the amino acid sequence 1174-but not intact TSP-1 - was able to stimulate tubulogenesis (Ferrari do Outeiro-Bernstein et al., 2002) when physically incorporated into fibrin plugs, a 3D support largely considered as a "provisional matrix" comparable to inflammatory edema and tumor microenvironments (Dvorak, 2015). The two sequences known for both containing affinity for GAGs (TSP-HepI, aa 1735 and TSP-HepII, aa 78-94) and destabilizing focal adhesions (Murphy-Ullrich et al., 1993) retained the major pro-angiogenic activity of HBD (Nunes et al., 2008). Competitive binding assays indicated that the two TSP-1 motifs could exert their effects by interfering with the recognition of the high-affinity C-terminal heparin-binding domain of FN (FN HepII) by cell surface SDC4. However, it is important to note that this interference in the action of FN did not affect the maintenance of cell viability, since pathways activated by SDC- 4 in its regular role in focal adhesions, e.g., its ability to promote the sequential activation of protein-kinase C- $\alpha$ (PKC- $\alpha$, a hallmark of SDC- 4 activation) and phosphoinositide 3-kinase (PI3K), which in turn activates Akt/protein-kinase B (PKB; Oh et al., 1997; Ilan et al., 1998), were also activated by the HBD domain and its angiogenic peptides TSP-HepI and TSP-HepII (Nunes et al., 2008). These data suggest that, in tissue remodeling microenvironments, the $\mathrm{N}$-terminal HBD possibly generated by proteases may provide the appropriate level of adhesion relaxation of endothelial cells engaged in tubulogenesis, while preserving cell viability.

Additional evidence for the biological relevance of proangiogenic activities of the HBD domain of TSP-1 came from studies performed with endothelial colony-forming cells (ECFC), or endothelial progenitor cells, isolated from human cord blood (Dias et al., 2012). Besides stimulating endothelial tubulogenesis of ECFC, as previously observed with adult primary endothelial cells (HUVECs; Nunes et al., 2008), TSPHepI peptide strongly potentiated FGF-2 angiogenic activity in vivo, in the Matrigel plug model.

Adhesion to established endothelial layers is one of the key steps of endothelial progenitor recruitment to ischemic/inflammatory sites. It was shown that the overnight pre-conditioning of ECFC with soluble TSP-HepI (but not with intact TSP-1) significantly increased the adhesion of progenitors to HUVEC monolayers under shear flow (Dias et al., 2012). Interestingly, pre-conditioning with TSP-HepI also resulted in augmented levels of $\alpha 6$ integrin chain on ECFC surfaces. The presence of a neutralizing anti-SDC-4 antibody during the preconditioning of ECFC with TSP-HepI inhibited their adhesion to HUVEC monolayers by $84 \%$. While the exact mechanisms for these effects remain to be explored, these data suggested a role of HBD domain in "priming" SDC- $4 / \alpha 6 \beta 1$ cooperation.

The study of the crystal structure of the HBD (amino acids 1240) and its complex with a synthetic pentameric heparin (Tan et al., 2008) has shown that, although the positively charged residues of TSP-HepI and TSP-HepII are well separated on the primary sequence of $\mathrm{HBD}$, they congregate to form a patch in 
the tertiary structure of the domain. Thus, these heparin-binding motifs used for SDC-4 recognition might be accessible in native $\mathrm{N}$-terminal fragments rapidly cleaved in vitro and in variable sizes by several proteases relevant to the vascular compartment (Bonnefoy and Legrand, 2000; Morandi, 2009).

\section{LRP1 AS A RECEPTOR FOR TSP-1}

The low density LRP1 is a large cell surface receptor that mediates the endocytosis of a number of different ligands including apolipoprotein E-enriched lipoproteins, protease inhibitor complexes, and matrix proteins including TSP-1 (Lillis et al., 2005). LRP1 is a member of the low-density lipoprotein (LDL) receptor family, which contains seven members that are closely related including the LDL receptor, very low density lipoprotein (VLDL) receptor, apoE receptor2, multiple epidermal growth factor-like domains 7 (MEGF7), glycoprotein 330 (gp330/megalin/LRP2), LRP1, and LRP1B. Similar to the other family members, LRP1 contains several modular structures including cysteine-rich complement-type repeats, EGF repeats, $\beta$-propeller domains, a transmembrane domain, and a cytoplasmic domain (Herz et al., 1990). LRP-1 is expressed by many tissues including liver, lung, and brain and in multiple cell types including hepatocytes, fibroblasts, smooth muscle cells, neurons, and macrophages (Moestrup et al., 1992).

Similar to the other family members, LRP1 is an endocytic receptor that at least partly functions to regulate the concentration of extracellular ligand by transporting these ligands through clathrin-coated pits into intracellular vesicles. LRP1 internalizes more than 40 ligands from the pericellular and extracellular environment, including proteinases, ECM proteins, growth factors, and cell surface receptors (Strickland et al., 2002).

Lipoprotein receptor-related protein 1 recognizes a vast number of different ligands and thus mechanisms must be present to inhibit premature association with ligands in the endoplasmic reticulum and allow for proper targeting to the plasma membrane. To facilitate this, the chaperone receptor associated protein (RAP) binds LRP1 with high affinity at multiple sites and antagonizes ligand binding while it is in the endoplasmic reticulum (Herz et al., 1991; Williams et al., 1992).

Lipoprotein receptor-related protein 1 binds TSP-1 at the $\mathrm{N}$-terminal heparin-binding domain, resulting in internalization and degradation in lysosomes (Chen et al., 1996). Through this internalization, TSP-1 can act as a bridging molecule between LRP1 and extracellular ligands and facilitate their clearance. TSP1/LRP-1 binding has been reported to be an important mechanism of clearing matrix metalloproteinases (MMPs) from the extracellular space (Bein and Simons, 2000; Yang et al., 2001). TSP-1 can also associate with cell surface proteoglycans and undergo endocytosis and degradation in an LRP-1-dependent fashion (Godyna et al., 1995; Mikhailenko et al., 1995). LRP-1 and TSP-1 can also act as co-receptors and in the presence of calreticulin can initiate a signaling cascade that results in focal adhesion disassembly (Orr et al., 2003). In the ovary, TSP-1 has been shown to participate in the clearance of VEGF via LRP1, reducing VEGF bioavailability and inhibiting ovarian follicular angiogenesis (Greenaway et al., 2007). TSP-1 also reduces the bioavailability of fibroblast growth factor 2 and hepatocyte growth factor/scatter factor (HGF/SF; Margosio et al., 2003).

\section{CD36 AS A RECEPTOR FOR TSP-1}

CD36 is a transmembrane glycoprotein that is a member of the class B scavenger receptor family. It functions as a long chain fatty acid translocase and a receptor for TSP-1, TSP-2, and collagen (Silverstein and Febbraio, 2009). Through these interactions, CD36 participates in a wide range of physiological processes, including fatty acid metabolism, atherosclerosis, and angiogenesis. CD36 mediates the inhibition of endothelial cell migration and proliferation by TSP-1. Significant quantities of CD36 are found in platelets, macrophages, adipocytes and endothelial cells (Silverstein and Febbraio, 2009). In general, CD36 is expressed on small, but not large, vessel endothelial cells (Luscinskas and Lawler, 1994). Whereas the expression of integrins on tumor vessels was found to vary with the stage of tumor progression in the Rip-Tag model of pancreatic cancer, CD36 is expressed at all stages (Xie et al., 2011). CD36 is a well characterized receptor for TSP-1 in endothelial cells (Jimenez et al., 2000). Engagement of CD36 by TSP-1 activates Fyn, JNK, and $\mathrm{p} 38 \mathrm{MAPK}$ to induce apoptosis through activation of caspase8- and -9-dependent pathways (Jimenez et al., 2000).

The membrane localization of CD36 and its mobility in the plasma membrane has been well studied. CD36 has been reported to be enriched in lipid rafts and tetraspaninenriched microdomains (Miao et al., 2001; Thorne et al., 2006). The tetraspanins are a family of proteins that contain four transmembrane domains (Hemler, 2014; van Deventer et al., 2020). They undergo homo- and heterotypic association to form distinct regions in the plasma membrane that are enriched in integrins and other membrane proteins. The $\mathrm{N}$ - and C-terminals of CD36 contain short intracellular sequences that do not contain consensus sequences for the docking of signal transduction proteins. Thus, CD36 is thought to initiate signal transduction indirectly through complex formation with lipids, integrins and itself. In platelets, the association of CD36 with the Src family kinase Lyn is reportedly mediated by lipids (Thorne et al., 2006). However, the specific Src family kinase that is immunoprecipitated with CD36 depends upon the presence of TSP-1 (Sun et al., 2009). In endothelial cells from wild-type mice, CD36 associates with Fyn and the quantity of Fyn that coimmunoprecipitates with CD36 increases when 3TSR is added. In TSP-1-null endothelial cells, Src replaces Fyn.

In 2006, Daviet et al. (1997) reported that CD36 exists as monomers and dimers in the plasma membrane. Whereas the addition of TSP-1 increases the formation of CD36 dimers in the membrane, TSP-1 did not induce dimerization of a soluble form of CD36. A more recent study reported that over-expression of CD36 in an immortalized endothelial cell line resulted in $40 \%$ of the CD36 molecules localizing to nanoclusters that contained as many as 75 CD36 molecules (Githaka et al., 2016). These nanoclusters are enriched in Fyn and F-actin. The size of the clusters and the phosphorylation of Fyn increases after addition of TSP-1 or an anti-CD36 IgM molecule (Githaka et al., 2016). The formation of 
nanoclusters is likely facilitated by the fact the CD36 mobility in the plasma membrane is restricted by the cytoskeleton (Jaqaman et al., 2011).

The clustering of CD36 promotes signal transduction through recruitment of other membrane proteins, such as integrins, and signaling molecules, such as Fyn and Syk (Figure 2; Kazerounian et al., 2011). Complex formation of CD36 with the tetraspanins CD9, CD81, and CD151 in platelets and endothelial cells has been reported (Miao et al., 2001; Kazerounian et al., 2011). In addition, association with $\alpha v, \alpha 5, \beta 1$, and $\beta 2$ integrins has been detected (Kazerounian et al., 2011). Since TSP-1 can engage integrins in the absence of CD36 (see below), it may form multivalent complexes with these proteins. In monocytes, the interaction of Syk with CD36 is reportedly mediated by integrins and FcR $\gamma$ (Heit et al., 2013).

In the context of angiogenesis, the association of CD36 with VEGF receptor 2 (VEGFR2) is a particularly intriguing one because it places receptors involved in pro- and antiangiogenic signaling in close proximity and raises the possibility that these complexes function as molecular angiogenic switches (Primo et al., 2005). Interestingly, this interaction is markedly reduced in the absence of TSP-1 (Primo et al., 2005; Zhang et al., 2009). VEGFR2 is a key receptor for the stimulation of endothelial cell proliferation and migration by VEGF during physiological and pathological angiogenesis (Simons et al., 2016). The close juxtaposition of a pro-angiogenic signaling receptor with CD36 offers a second pathway through which TSP-1 can antagonize angiogenesis. This pathway involves the recruitment of the phosphatase SHP1 to the CD36/VEGFR2 complex (Chu et al., 2013). SHP1 dephosphorylates VEGFR2 and suppresses the recruitment of pro-angiogenic, downstream signaling proteins. Addition of TSP-1 increases the quantity of SHP1 associated with VEGFR2 in endothelial and ovarian cancer cells (Russell et al., 2015). The inhibition of VEGFR2 phosphorylation by TSP-1 is disrupted when CD36 is mutated so as to lose its ability to interact with $\beta 1$ integrins (Primo et al., 2005).

Taken together, the data indicate that CD36 forms complexes with multiple other membrane proteins to affect endothelial behavior. Extracellular TSP-1 affects endothelial cell response to stimuli by modulating the composition of these complexes. The complexes that form may be heterogeneous in their composition and size.

\section{INTEGRIN BINDING TO TSP-1}

The integrins are a family of heterodimeric membrane proteins that function as receptors for extracellular matrix molecules, including collagens, FN, vitronectin, and laminins (Hynes, 2002; Luo et al., 2007). The term integrin was coined to reflect the integral role of these protein complexes as mediators of communication between the extracellular matrix and the cytoskeleton (Tamkun et al., 1986). In addition, a subset of integrins can activate TGF $\beta$ (Munger et al., 1999; Mu et al., 2002). An early comparison of platelet GPIIb/GPIIIa and endothelial cell $\alpha \mathrm{V} \beta 3$ was one of the first indications that integrins are an extensive family of proteins, with various members expressed on virtually all cell types (Charo et al., 1986). Integrins are differentially expressed on large vessel and microvascular endothelial cells, and by various growth factors and cytokines (Luscinskas and Lawler, 1994). As indicated above, integrins are included in tetraspanin-enriched microdomains where they can associate with CD36 and VEGFR-2 in a TSP1 -dependent manner. The $\alpha \mathrm{v}, \alpha 5, \beta 1$, and $\beta 2$ integrins have been reported to be present in these complexes (Hemler, 2014; van Deventer et al., 2020).

A direct RGD-dependent interaction of TSP- 1 with $\alpha v \beta 3$ has been reported (Lawler et al., 1988; Chen et al., 2000). The RGD sequence of TSP-1 lies within the type 3 repeats, which are a contiguous set of calcium-binding sites. Removal of calcium results in a significant change in the conformation of the TSP1 molecule and increases exposure of the RGD sequence (Sun et al., 1992). The structure of the type 3 repeats is stabilized by calcium and intrachain disulfide bonds (Tan et al., 2002). Sun et al. (1992) reported that partial reduction of the disulfide bonds in TSP-1 increases the exposure of the RGD sequence. A follow-up study by Hotchkiss et al. (1998) found a disulfide bond isomerase at the plasma membrane of endothelial cells that increased the accessibility of the RGD sequence of TSP1. Microvascular endothelial cell attachment to and chemotaxis toward TSP-1 are inhibited by antibodies to $\beta 3$ (Lawler et al., 1988; Taraboletti et al., 1990). TSP-1 may serve as a substrate for endothelial $\alpha v \beta 3$ in wounds where high quantities of TSP-1 are deposited by activated platelets.

TSP-1 inhibits the migration of human umbilical vein endothelial cells, which lack CD36, through an integrin $\beta 1$ dependent mechanism (Short et al., 2005). This pathway for inhibition of migration appears to involve PI3K. Interestingly, an antibody to $\beta 1$ integrin also inhibits VEGF-induced migration of microvascular endothelial cells that do express CD36 in the presence of an anti-CD36 antibody (Short et al., 2005). These data underscore the close interaction between CD36 and integrins that may arise from their physical proximity in the plasma membrane.

As described above, the $\mathrm{NH}_{2}$ domain of TSP-1 promotes angiogenesis through SDC-4 (Ferrari do Outeiro-Bernstein et al., 2002; Dias et al., 2012). This domain has also been reported to enhance angiogenesis through the $\alpha 9 \beta 1$ integrin on microvascular endothelial cells through pathways that involve Erk1/2 and paxillin (Staniszewska et al., 2007). The $\alpha 3 \beta 1$ and $\alpha 4 \beta 1$ integrins have also been reported to bind to the $\mathrm{NH}_{2}$ domain of TSP-1 (Chandrasekaran et al., 2000; Calzada et al., 2004). The binding of $\alpha 4 \beta 1$ to TSP- 1 was observed with venous but not microvascular endothelial cells (Calzada et al., 2004). The interaction of $\alpha 3 \beta 1$ and $\alpha 4 \beta 1$ integrins with TSP-1 reportedly promotes angiogenesis.

\section{CD47 AS A RECEPTOR FOR TSP-1}

CD47 is a $50 \mathrm{kDa}$ transmembrane receptor, also known as integrin-associated protein (IAP), that was initially identified as a protein lost from red blood cells in patients with $\mathrm{Rh}$ null hemolytic anemia (Miller et al., 1987). CD47 consists of an extracellular $\mathrm{N}$-terminal $\mathrm{IgV}$ domain, five transmembrane 

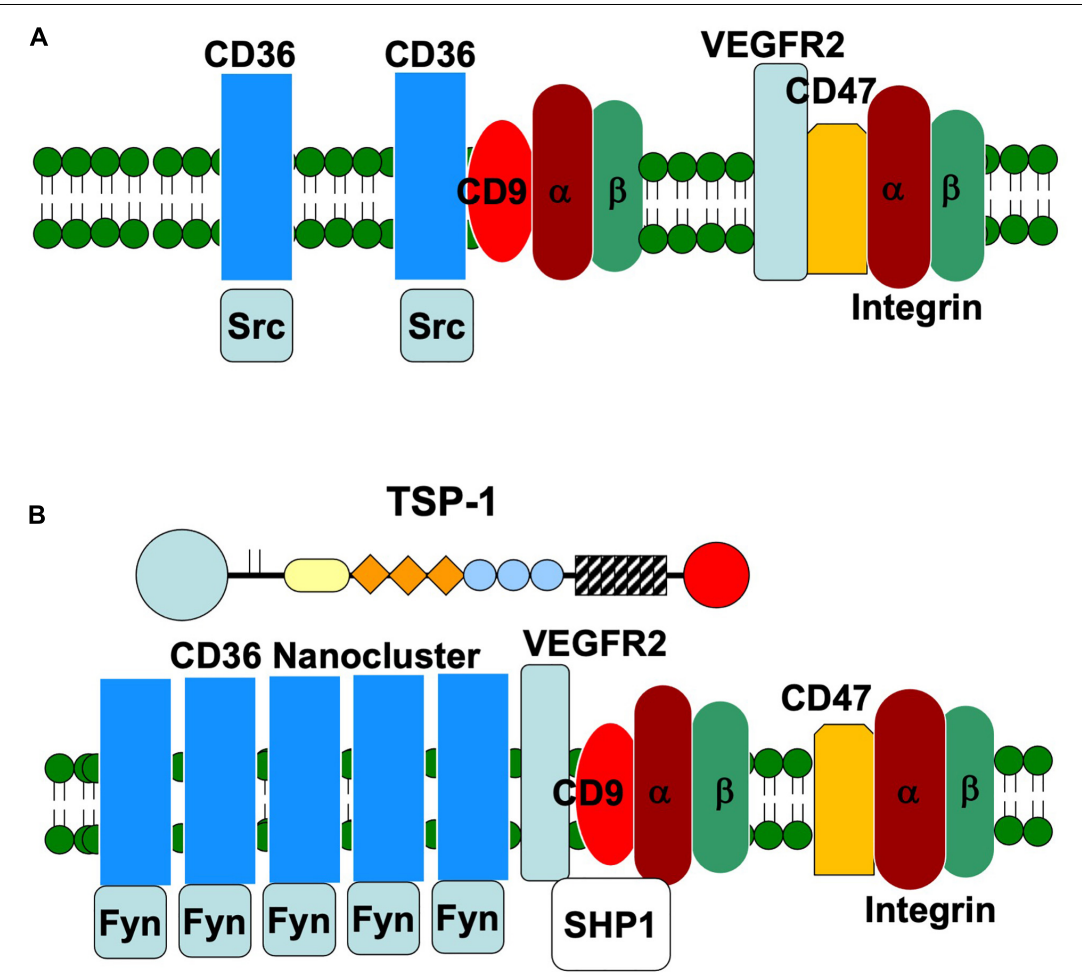

FIGURE 2 | Schematic representation of the organization of endothelial membrane proteins in the absence (A) and presence (B) of TSP-1. In the absence of TSP-1, Src co-immunoprecipitates with CD36. Fyn is preferentially associated with CD36 in the presence of TSP-1 and the formation of CD36 nanoclusters amplifies Fyn-mediated signal transduction. The presence of TSP-1 also promotes the dissociation of VEGFR2 from CD47 and the association of VEGFR2 with CD36. Integrins and SHP1 also complex with VEGFR2.

domains, and a short C-terminal intracellular tail (Mawby et al., 1994). Four alternatively spliced isoforms of CD47 exist, differing in the length of their cytoplasmic tails (Reinhold et al., 1995). CD47 has two main roles: as a ligand for the signal-regulatory protein alpha (SIRP $\alpha)$ and as a receptor for TSP-1. The CD47$\operatorname{SIRP} \alpha$ axis delivers inhibitory signals for phagocytosis and conveys a "don't eat me" signal that has important functions in hematopoiesis and innate immune surveillance (Tsai et al., 2010; McCracken et al., 2015; Horrigan and Reproducibility Project: Cancer Biology, 2017). Initial studies reported that CD47 is bound by TSP-1 at two peptide motifs on the carboxyterminal domain (Gao et al., 2017). While partial conservation of some of these residues is found in TSP-2 and -4, CD47 only binds to TSP-1 with high affinity (Isenberg et al., 2009). Some molecules of CD47 contain proteoglycan side chains that are important for high-affinity TSP-1 binding and signaling (Kaur et al., 2011). The interaction with the proteoglycan moieties may be specific to TSP-1.

Thrombospondin 1 binding to CD47 has an inhibitory influence on VEGF signaling. CD47 constitutively associates with VEGFR2 on endothelial (Kaur et al., 2010) and tumor (Russell et al., 2015) cells. Binding of CD47 by TSP-1 inhibits the association between CD47 and VEGFR-2 and disrupts VEGFR2 phosphorylation (Kaur et al., 2010). Ligation by TSP-1 prevents VEGFR2 autophosphorylation, which decreases activation of endothelial nitric oxide synthase (eNOS) associated with Akt phosphorylation. This is consistent with the increased Akt phosphorylation seen in retinal vasculature (Sun et al., 2009) and in ovarian peri-follicular vasculature (Greenaway et al., 2007) of TSP-1 null mice. Similar to the receptor clustering seen with CD36, recent evidence suggests that clustering of CD47 is also required for the high-affinity binding of TSP-1 (Wang et al., 2020). These results suggest that in addition to the amount of CD47 available, the distribution pattern of the receptor is important in regulating TSP-1 signaling.

Activation of CD47 by TSP-1 ultimately inhibits $\mathrm{Ca}^{2+} /$ calmodulin-mediated activation of eNOS (Bauer et al., 2010), activation of soluble guanylate cyclase (sGC) by nitric oxide (NO; Isenberg et al., 2006), and downstream activation of cGMP-dependent protein kinase (Isenberg et al., 2008b). At physiologic concentrations of TSP-1 (100-200 pM), CD47 is thought to be the dominant receptor for the ligand in inhibiting activation of soluble guanylate cyclase or cGMP-dependent protein kinase (Isenberg et al., 2006, 2008b). Using a systems biology approach, it was determined that enhancing binding of TSP-1 to CD47 decreases the amount of unbound TSP-1 and protects TSP-1 from cleavage, enhancing the anti-angiogenic effect of the ligand (Rohrs et al., 2016).

By disrupting TSP-1/CD47 signaling, angiogenesis can be enhanced, which can have therapeutic benefit. Both TSP-1 and CD47 null mice have decreased necrosis and improved healing in the cutaneous flap model and in full-thickness skin grafts. 
Skin grafts implanted on wild-type mice fail due to a lack of angiogenesis and tissue perfusion, while those grafted onto TSP1 or CD47 null mice survive (Isenberg et al., 2008a). Similarly, pancreatic islet grafts have improved survival in TSP-1 null mice, due to increased angiogenesis (Olerud et al., 2008). Using function blocking antibodies to TSP-1 or CD47 has also been shown to reestablish perfusion in ischemic tissues in rodents and pigs (Isenberg et al., 2007, 2008a). In cancer, CD47 null mice had elevated VEGF and VEGFR2 expression and there was increased angiogenesis and accelerated tumor progression in a syngeneic murine model of prostate cancer (Gao et al., 2017).

In addition to its role in angiogenesis, TSP-1/CD47 binding is also involved in regulating tumor immunity. Ligation of CD47 on immune cells inactivates antitumor adaptive immunosurveillance and directly inhibits TCR-mediated T cell activation (Li et al., 2001, 2002). Through CD47, TSP-1 also reduces VEGF-induced immunosuppression in tumors (Kaur et al., 2014), and enhances antitumor immunity by stimulating CD8+cytotoxic T cells (Soto-Pantoja et al., 2014). TSP-1 signaling via CD47 also regulate $\mathrm{NK}$ and $\mathrm{DC}$ functions that impact adaptive immunity (Weng et al., 2014).

\section{CONCLUSION AND FUTURE DIRECTIONS}

In this review, we have highlighted the interaction of TSP-1 with endothelial membrane proteins (Figure 1). Through these many interactions, TSP-1 is able to direct the formation of supramolecular complexes that vary in composition over time to modulate the endothelial cell's response to environmental stimuli (Kazerounian and Lawler, 2018). The data indicate that endothelial cell behavior is determined at the level of the plasma membrane and thus early in the signal generation. The remodeling of membrane protein complexes that form in response to TSP-1 serves to integrate signals from pro- and anti-angiogenic stimuli. In the presence of TSP-1, VEGFR2 dissociates from CD47 and complexes with CD36, which results in enhanced association with integrins and SHP1, leading to dephosphorylation of VEGFR2 and suppression of VEGF signal transduction. TSP-1 also initiates an anti-angiogenic signal through CD36 that leads to decreased endothelial cell migration and apoptosis. This pathway is amplified by the ability of TSP-1 to drive the formation of CD36 nanoclusters, which in turn cluster Fyn. It is important to determine how the concentrations of TSP1 and VEGF affect the above processes. Does the quantity of the various supramolecular complexes have a linear effect on the net

\section{REFERENCES}

Adams, J. C., and Lawler, J. (2011). The thrombospondins. Cold Spring Harb. Perspect. Biol. 3:a009712.

Agah, A., Kyriakides, T. R., Lawler, J., and Bornstein, P. (2002). The lack of thrombospondin-1 (TSP1) dictates the course of wound healing in doubleTSP1/TSP2-null mice. Am. J. Pathol. 161, 831-839. doi: 10.1016/s00029440(10)64243-5 signal, or are there thresholds that must be exceeded in order to initiate response in a stepwise fashion? Can the negative signal elicited by TSP-1 be reversed at some stages and not at others? In addition, our understanding of the role of integrins and their ligands in the way in which endothelial cells respond to TSP-1 is far from complete.

Since tumor growth experiments in TSP-1-null mice indicate that the absence of the intact molecule leads to increased angiogenesis, the pro-angiogenic activity of the $\mathrm{NH}_{2}$ domain may be masked or outweighed by the anti-angiogenic activity of the TSRs and the C-terminal domain (Lawler et al., 2001; Xie et al., 2011). In addition, the type 3 repeats have been reported to inhibit angiogenesis by binding to fibroblast growth factor 2 (FGF2; Colombo et al., 2010). Proteolytic cleavage of TSP1 may produce pro- and anti-angiogenic domains with distinct biological properties. Indeed, the first study to show that TSP-1 is anti-angiogenic detected a proteolytic fragment of TSP-1 in the condition media of p53 deficient fibroblasts from Li-Fraumeni patients (Dameron et al., 1994). This fragment was produced by a proteolytic cleavage between the HBD and the TSRs. We need to better understand the physiological conditions that lead to the release of the HBD from the intact molecule and the disposition of the two or more fragments that are produced. Do the two fragments have different biodistribution and/or stability? Like the intact molecule, the HBD has multiple ligands, including proteoglycans, LPR1, calreticulin and integrins.

\section{AUTHOR CONTRIBUTIONS}

All authors listed have made a substantial, direct and intellectual contribution to the work, and approved it for publication.

\section{FUNDING}

This work was supported by operating grants to JP from the Canadian Institutes for Health Research, Cancer Research Society, and Ovarian Cancer Canada. The project was also supported by a CAO Pilot Grant from the Beth Israel Deaconess Medical Center. VM received supports from the Brazilian federal agencies CAPES and CNPq, and from FAPERJ (a public funding organism of the Rio de Janeiro State).

\section{ACKNOWLEDGMENTS}

We thank Sami Lawler for editing the text.

Balint, S., Muller, S., Fischer, R., Kessler, B. M., Harkiolaki, M., Valitutti, S., et al. (2020). Supramolecular attack particles are autonomous killing entities released from cytotoxic T cells. Science. 368, 897-901. doi: 10.1126/science.aay 9207

Bauer, E. M., Qin, Y., Miller, T. W., Bandle, R. W., Csanyi, G., Pagano, P. J., et al. (2010). Thrombospondin-1 supports blood pressure by limiting eNOS activation and endothelial-dependent vasorelaxation. Cardiovasc. Res. 88, 471481. doi: $10.1093 / \mathrm{cvr} / \mathrm{cvq} 218$ 
Bein, K., and Simons, M. (2000). Thrombospondin type 1 repeats interact with matrix metalloproteinase 2. Regulation of metalloproteinase activity. J. Biol. Chem. 275, 32167-32173. doi: 10.1074/jbc.m003834200

Bonnefoy, A., and Legrand, C. (2000). Proteolysis of subendothelial adhesive glycoproteins (fibronectin, thrombospondin, and von Willebrand factor) by plasmin, leukocyte cathepsin G, and elastase. Thromb. Res. 98, 323-332. doi: 10.1016/s0049-3848(99)00242-x

Calzada, M. J., Zhou, L., Sipes, J. M., Zhang, J., Krutzsch, H. C., Iruela-Arispe, M. L., et al. (2004). Alpha4betal integrin mediates selective endothelial cell responses to thrombospondins 1 and 2 in vitro and modulates angiogenesis in vivo. Circ. Res. 94, 462-470. doi: 10.1161/01.res.0000115555.05668.93

Chandrasekaran, L., He, C. Z., Al-Barazi, H., Krutzsch, H. C., Iruela-Arispe, M. L., and Roberts, D. D. (2000). Cell contact-dependent activation of alpha3betal integrin modulates endothelial cell responses to thrombospondin-1. Mol. Biol. Cell. 11, 2885-2900. doi: 10.1091/mbc.11.9.2885

Charo, I. F., Fitzgerald, L. A., Steiner, B., Rall, S. C. Jr., Bekeart, L. S., and Phillips, D. R. (1986). Platelet glycoproteins IIb and IIIa: evidence for a family of immunologically and structurally related glycoproteins in mammalian cells. Proc. Natl. Acad. Sci. U.S.A. 83, 8351-8355. doi: 10.1073/pnas.83.21.8351

Chen, H., Herndon, M. E., and Lawler, J. (2000). The cell biology of thrombospondin-1. Matrix Biol. 19, 597-614. doi: 10.1016/s0945-053x(00) 00107-4

Chen, H., Sottile, J., Strickland, D. K., and Mosher, D. F. (1996). Binding and degradation of thrombospondin-1 mediated through heparan sulphate proteoglycans and low-density-lipoprotein receptor-related protein: localization of the functional activity to the trimeric N-terminal heparinbinding region of thrombospondin-1. Biochem. J. 318( $\mathrm{Pt} 3)$, 959-963. doi: 10.1042/bj3180959

Chu, L. Y., Ramakrishnan, D. P., and Silverstein, R. L. (2013). Thrombospondin-1 modulates VEGF signaling via CD36 by recruiting SHP-1 to VEGFR2 complex in microvascular endothelial cells. Blood. 122, 1822-1832. doi: 10.1182/blood2013-01-482315

Chung, H., Multhaupt, H. A., Oh, E. S., and Couchman, J. R. (2016). Minireview: syndecans and their crucial roles during tissue regeneration. FEBS Lett. 590, 2408-2417. doi: 10.1002/1873-3468.12280

Clezardin, P., Lawler, J., Amiral, J., Quentin, G., and Delmas, P. (1997). Identification of cell adhesive active sites in the $\mathrm{N}$-terminal domain of thrombospondin-1. Biochem. J. 321(Pt 3), 819-827. doi: 10.1042/bj3210819

Colombo, G., Margosio, B., Ragona, L., Neves, M., Bonifacio, S., Annis, D. S., et al. (2010). Non-peptidic thrombospondin-1 mimics as fibroblast growth factor-2 inhibitors: an integrated strategy for the development of new antiangiogenic compounds. J. Biol. Chem. 285, 8733-8742. doi: 10.1074/jbc.m109.085605

Corless, C. L., Mendoza, A., Collins, T., and Lawler, J. (1992). Colocalization of thrombospondin and syndecan during murine development. Dev. Dyn. 193, 346-358. doi: 10.1002/aja.1001930408

Couchman, J. R. (2003). Syndecans: proteoglycan regulators of cell-surface microdomains? Nat. Rev. Mol. Cell Biol. 4, 926-937. doi: 10.1038/nrm1257

Dameron, K. M., Volpert, O. V., Tainsky, M. A., and Bouck, N. (1994). Control of angiogenesis in fibroblasts by p53 regulation of thrombospondin-1. Science. 265, 1582-1584. doi: 10.1126/science.7521539

Daviet, L., Malvoisin, E., Wild, T. F., and McGregor, J. L. (1997). Thrombospondin induces dimerization of membrane-bound, but not soluble CD36. Thromb. Haemost. 78, 897-901. doi: 10.1055/s-0038-1657649

De Rossi, G., and Whiteford, J. R. (2014). Syndecans in angiogenesis and endothelial cell biology. Biochem. Soc. Trans. 42, 1643-1646. doi: 10.1042/ bst20140232

Dias, J. V., Benslimane-Ahmim, Z., Egot, M., Lokajczyk, A., Grelac, F., GalyFauroux, I., et al. (2012). A motif within the N-terminal domain of TSP-1 specifically promotes the proangiogenic activity of endothelial colony-forming cells. Biochem. Pharmacol. 84, 1014-1023. doi: 10.1016/j.bcp.2012.07.006

Dvorak, H. F. (2015). Tumors: wounds that do not heal-redux. Cancer Immunol. Res. 3, 1-11. doi: 10.1158/2326-6066.cir-14-0209

Echtermeyer, F., Streit, M., Wilcox-Adelman, S., Saoncella, S., Denhez, F., Detmar, M., et al. (2001). Delayed wound repair and impaired angiogenesis in mice lacking syndecan-4. J. Clin. Invest. 107, R9-R14. doi: 10.1007/978-3-64227814-3_85-2

Feitsma, K., Hausser, H., Robenek, H., Kresse, H., and Vischer, P. (2000). Interaction of thrombospondin-1 and heparan sulfate from endothelial cells.
Structural requirements of heparan sulfate. J. Biol. Chem. 275, 9396-9402. doi: $10.1074 /$ jbc.275.13.9396

Ferrari do Outeiro-Bernstein, M. A., Nunes, S. S., Andrade, A. C., Alves, T. R., Legrand, C., and Morandi, V. (2002). A recombinant NH(2)-terminal heparinbinding domain of the adhesive glycoprotein, thrombospondin-1, promotes endothelial tube formation and cell survival: a possible role for syndecan-4 proteoglycan. Matrix Biol. 21, 311-324. doi: 10.1016/s0945-053x(02)00010-0

Frangogiannis, N. G., Ren, G., Dewald, O., Zymek, P., Haudek, S., Koerting, A., et al. (2005). Critical role of endogenous thrombospondin-1 in preventing expansion of healing myocardial infarcts. Circulation. 111, 2935-2942. doi: 10.1161/circulationaha.104.510354

Gao, L., Chen, K., Gao, Q., Wang, X., Sun, J., and Yang, Y. G. (2017). CD47 deficiency in tumor stroma promotes tumor progression by enhancing angiogenesis. Oncotarget 8, 22406-22413. doi: 10.18632/oncotarget.9899

Garcia-Parajo, M. F., Cambi, A., Torreno-Pina, J. A., Thompson, N., and Jacobson, K. (2014). Nanoclustering as a dominant feature of plasma membrane organization. J. Cell Sci. 127(Pt 23), 4995-5005. doi: 10.1242/jcs.146340

Githaka, J. M., Vega, A. R., Baird, M. A., Davidson, M. W., Jaqaman, K., and Touret, N. (2016). Ligand-induced growth and compaction of CD36 nanoclusters enriched in Fyn induces Fyn signaling. J. Cell Sci. 129, 4175-4189. doi: 10.1242/ jcs. 188946

Godyna, S., Liau, G., Popa, I., Stefansson, S., and Argraves, W. S. (1995). Identification of the low density lipoprotein receptor-related protein (LRP) as an endocytic receptor for thrombospondin-1. J. Cell Biol. 129, 1403-1410. doi: $10.1083 /$ jcb.129.5.1403

Gondelaud, F., and Ricard-Blum, S. (2019). Structures and interactions of syndecans. FEBS J. 286, 2994-3007. doi: 10.1111/febs.14828

Greenaway, J., Lawler, J., Moorehead, R., Bornstein, P., Lamarre, J., and Petrik, J. (2007). Thrombospondin-1 inhibits VEGF levels in the ovary directly by binding and internalization via the low density lipoprotein receptorrelated protein-1 (LRP-1). J. Cell Physiol. 210, 807-818. doi: 10.1002/jcp. 20904

Guo, N. H., Krutzsch, H. C., Negre, E., Vogel, T., Blake, D. A., and Roberts, D. D. (1992). Heparin- and sulfatide-binding peptides from the type I repeats of human thrombospondin promote melanoma cell adhesion. Proc. Natl. Acad. Sci. U.S.A. 89, 3040-3044. doi: 10.1073/pnas.89.7.3040

Heit, B., Kim, H., Cosio, G., Castano, D., Collins, R., Lowell, C. A., et al. (2013). Multimolecular signaling complexes enable Syk-mediated signaling of CD36 internalization. Dev. Cell. 24, 372-383. doi: 10.1016/j.devcel.2013.01.007

Hemler, M. E. (2014). Tetraspanin proteins promote multiple cancer stages. Nat. Rev. Cancer 14, 49-60. doi: 10.1038/nrc3640

Herz, J., Goldstein, J. L., Strickland, D. K., Ho, Y. K., and Brown, M. S. (1991). $39-\mathrm{kDa}$ protein modulates binding of ligands to low density lipoprotein receptor-related protein/alpha 2-macroglobulin receptor. J. Biol. Chem. 266, 21232-21238. doi: 10.1016/s0021-9258(18)54845-6

Herz, J., Kowal, R. C., Goldstein, J. L., and Brown, M. S. (1990). Proteolytic processing of the $600 \mathrm{kd}$ low density lipoprotein receptor-related protein (LRP) occurs in a trans-Golgi compartment. EMBO J. 9, 1769-1776. doi: 10.1002/j. 1460-2075.1990.tb08301.x

Horrigan, S. K., and Reproducibility Project: Cancer Biology (2017). Replication study: the CD47-signal regulatory protein alpha (SIRPa) interaction is a therapeutic target for human solid tumors. Elife 6:e18173.

Hotchkiss, K. A., Matthias, L. J., and Hogg, P. J. (1998). Exposure of the cryptic Arg-Gly-Asp sequence in thrombospondin-1 by protein disulfide isomerase. Biochim. Biophys. Acta. 1388, 478-488. doi: 10.1016/s0167-4838(98)00211-8

Hynes, R. O. (2002). Integrins: bidirectional, allosteric signaling machines. Cell. $110,673-687$.

Ilan, N., Mahooti, S., and Madri, J. A. (1998). Distinct signal transduction pathways are utilized during the tube formation and survival phases of in vitro angiogenesis. J. Cell Sci. 111(Pt 24), 3621-3631.

Isenberg, J. S., Annis, D. S., Pendrak, M. L., Ptaszynska, M., Frazier, W. A., Mosher, D. F., et al. (2009). Differential interactions of thrombospondin-1, -2 , and -4 with CD47 and effects on cGMP signaling and ischemic injury responses. J. Biol. Chem. 284, 1116-1125. doi: 10.1074/jbc.m804860200

Isenberg, J. S., Ridnour, L. A., Dimitry, J., Frazier, W. A., Wink, D. A., and Roberts, D. D. (2006). CD47 is necessary for inhibition of nitric oxide-stimulated vascular cell responses by thrombospondin-1. J. Biol. Chem. 281, 26069-26080. doi: 10.1074/jbc.m605040200 
Isenberg, J. S., Romeo, M. J., Abu-Asab, M., Tsokos, M., Oldenborg, A., Pappan, L., et al. (2007). Increasing survival of ischemic tissue by targeting CD47. Circ. Res. 100, 712-720. doi: 10.1161/01.res.0000259579.35787.4e

Isenberg, J. S., Romeo, M. J., Maxhimer, J. B., Smedley, J., Frazier, W. A., and Roberts, D. D. (2008a). Gene silencing of CD47 and antibody ligation of thrombospondin-1 enhance ischemic tissue survival in a porcine model: implications for human disease. Ann. Surg. 247, 860-868. doi: 10.1097/sla. 0b013e31816c4006

Isenberg, J. S., Romeo, M. J., Yu, C., Yu, C. K., Nghiem, K., Monsale, J., et al. (2008b). Thrombospondin-1 stimulates platelet aggregation by blocking the antithrombotic activity of nitric oxide/cGMP signaling. Blood. 111, 613-623. doi: 10.1182/blood-2007-06-098392

Jaqaman, K., Kuwata, H., Touret, N., Collins, R., Trimble, W. S., Danuser, G., et al. (2011). Cytoskeletal control of CD36 diffusion promotes its receptor and signaling function. Cell. 146, 593-606. doi: 10.1016/j.cell.2011.06.049

Jimenez, B., Volpert, O. V., Crawford, S. E., Febbraio, M., Silverstein, R. L., and Bouck, N. (2000). Signals leading to apoptosis-dependent inhibition of neovascularization by thrombospondin-1. Nat. Med. 6, 41-48. doi: 10.1038/ 71517

Kaur, S., Chang, T., Singh, S. P., Lim, L., Mannan, P., Garfield, S. H., et al. (2014). CD47 signaling regulates the immunosuppressive activity of VEGF in T cells. J. Immunol. 193, 3914-3924. doi: 10.4049/jimmunol.1303116

Kaur, S., Kuznetsova, S. A., Pendrak, M. L., Sipes, J. M., Romeo, M. J., Li, Z., et al. (2011). Heparan sulfate modification of the transmembrane receptor CD47 is necessary for inhibition of $\mathrm{T}$ cell receptor signaling by thrombospondin-1. J. Biol. Chem. 286, 14991-15002. doi: 10.1074/jbc.m110.179663

Kaur, S., Martin-Manso, G., Pendrak, M. L., Garfield, S. H., Isenberg, J. S., and Roberts, D. D. (2010). Thrombospondin-1 inhibits VEGF receptor-2 signaling by disrupting its association with CD47. J. Biol. Chem. 285, 38923-38932. doi: $10.1074 /$ jbc.m110.172304

Kazerounian, S., and Lawler, J. (2018). Integration of pro- and anti-angiogenic signals by endothelial cells. J. Cell Commun. Signal. 12, 171-179. doi: 10.1007/ s12079-017-0433-3

Kazerounian, S., Duquette, M., Reyes, M. A., Lawler, J. T., Song, K., Perruzzi, C., et al. (2011). Priming of the vascular endothelial growth factor signaling pathway by thrombospondin-1, CD36, and spleen tyrosine kinase. Blood. 117, 4658-4666. doi: 10.1182/blood-2010-09-305284

Lawler, J. W., Slayter, H. S., and Coligan, J. E. (1978). Isolation and characterization of a high molecular weight glycoprotein from human blood platelets. J. Biol. Chem. 253, 8609-8616. doi: 10.1016/s0021-9258(17)34336-3

Lawler, J., Miao, W. M., Duquette, M., Bouck, N., Bronson, R. T., and Hynes, R. O. (2001). Thrombospondin-1 gene expression affects survival and tumor spectrum of p53-deficient mice. Am. J. Pathol. 159, 1949-1956. doi: 10.1016/ s0002-9440(10)63042-8

Lawler, J., Weinstein, R., and Hynes, R. O. (1988). Cell attachment to thrombospondin: the role of ARG-GLY-ASP, calcium, and integrin receptors. J. Cell Biol. 107(6 Pt 1), 2351-2361. doi: 10.1083/jcb.107.6.2351

Li, S. S., Ivanoff, A., Bergstrom, S. E., Sandstrom, A., Christensson, B., van Nerven, J., et al. (2002). T lymphocyte expression of thrombospondin-1 and adhesion to extracellular matrix components. Eur. J. Immunol. 32, 1069-1079. doi: 10.1002/1521-4141(200204)32:4<1069::aid-immu1069>3.0.co;2-e

Li, Z., He, L., Wilson, K., and Roberts, D. (2001). Thrombospondin-1 inhibits TCR-mediated T lymphocyte early activation. J Immunol. 166, 2427-2436. doi: 10.4049/jimmunol.166.4.2427

Lillis, A. P., Mikhailenko, I., and Strickland, D. K. (2005). Beyond endocytosis: LRP function in cell migration, proliferation and vascular permeability. J. Thromb. Haemost. 3, 1884-1893. doi: 10.1111/j.1538-7836.2005.01371.x

Luo, B. H., Carman, C. V., and Springer, T. A. (2007). Structural basis of integrin regulation and signaling. Annu. Rev. Immunol. 25, 619-647. doi: 10.1146/ annurev.immunol.25.022106.141618

Luscinskas, F. W., and Lawler, J. (1994). Integrins as dynamic regulators of vascular function. FASEB J. 8, 929-938. doi: 10.1096/fasebj.8.12.7522194

Margosio, B., Marchetti, D., Vergani, V., Giavazzi, R., Rusnati, M., Presta, M., et al. (2003). Thrombospondin 1 as a scavenger for matrix-associated fibroblast growth factor 2. Blood. 102, 4399-4406. doi: 10.1182/blood-2003-03-0893

Matsui, Y., Ikesue, M., Danzaki, K., Morimoto, J., Sato, M., Tanaka, S., et al. (2011). Syndecan-4 prevents cardiac rupture and dysfunction after myocardial infarction. Circ. Res. 108, 1328-1339. doi: 10.1161/circresaha.110.235689
Mawby, W. J., Holmes, C. H., Anstee, D. J., Spring, F. A., and Tanner, M. J. (1994). Isolation and characterization of CD47 glycoprotein: a multispanning membrane protein which is the same as integrin-associated protein (IAP) and the ovarian tumour marker OA3. Biochem. J. 304(Pt 2), 525-530. doi: 10.1042/ bj3040525

McCracken, M. N., Cha, A. C., and Weissman, I. L. (2015). Molecular pathways: activating T cells after cancer cell phagocytosis from blockade of CD47 "Don't Eat Me" signals. Clin. Cancer Res. 21, 3597-3601. doi: 10.1158/1078-0432.ccr14- 2520

Merle, B., Malaval, L., Lawler, J., Delmas, P., and Clezardin, P. (1997). Decorin inhibits cell attachment to thrombospondin-1 by binding to a KKTR-dependent cell adhesive site present within the N-terminal domain of thrombospondin1. J. Cell Biochem. 67, 75-83. doi: 10.1002/(sici)1097-4644(19971001)67:1<75:: aid-jcb8 $>3.0 . \operatorname{co} ; 2-\mathrm{t}$

Miao, W. M., Vasile, E., Lane, W. S., and Lawler, J. (2001). CD36 associates with CD9 and integrins on human blood platelets. Blood 97, 1689-1696. doi: 10. 1182/blood.v97.6.1689

Michaely, P., Li, W. P., Anderson, R. G., Cohen, J. C., and Hobbs, H. H. (2004). The modular adaptor protein ARH is required for low density lipoprotein (LDL) binding and internalization but not for LDL receptor clustering in coated pits. J. Biol. Chem. 279, 34023-34031. doi: 10.1074/jbc.m405242200

Mikhailenko, I., Kounnas, M. Z., and Strickland, D. K. (1995). Low density lipoprotein receptor-related protein/alpha 2-macroglobulin receptor mediates the cellular internalization and degradation of thrombospondin. A process facilitated by cell-surface proteoglycans. J. Biol. Chem. 270, 9543-9549. doi: $10.1074 /$ jbc. 270.16 .9543

Miller, Y. E., Daniels, G. L., Jones, C., and Palmer, D. K. (1987). Identification of a cell-surface antigen produced by a gene on human chromosome 3 (cen-q22) and not expressed by Rhnull cells. Am. J. Hum. Genet. 41, 1061-1070.

Moestrup, S. K., Gliemann, J., and Pallesen, G. (1992). Distribution of the alpha 2 -macroglobulin receptor/low density lipoprotein receptor-related protein in human tissues. Cell. Tissue Res. 269, 375-382. doi: 10.1007/bf00353892

Morandi, V. (2009). The N-terminal domain of thrombospondin-1: a key for the dual effect of TSP-1 in angiogenesis and cancer progression? ScientificWorldJournal. 9, 133-136. doi: 10.1100/tsw.2009.11

$\mathrm{Mu}, \mathrm{D}$., Cambier, S., Fjellbirkeland, L., Baron, J. L., Munger, J. S., Kawakatsu, H., et al. (2002). The integrin alpha(v)beta8 mediates epithelial homeostasis through MT1-MMP-dependent activation of TGF-beta1. J. Cell Biol. 157, 493507. doi: $10.1083 /$ jcb. 200109100

Munger, J. S., Huang, X., Kawakatsu, H., Griffiths, M. J., Dalton, S. L., Wu, J., et al. (1999). The integrin alpha $\mathrm{v}$ beta 6 binds and activates latent TGF beta 1: a mechanism for regulating pulmonary inflammation and fibrosis. Cell 96, 319-328. doi: 10.1016/s0092-8674(00)80545-0

Munoz, E. M., and Linhardt, R. J. (2004). Heparin-binding domains in vascular biology. Arterioscler. Thromb. Vasc. Biol. 24, 1549-1557. doi: 10.1161/01.atv. $0000137189.22999 .3 \mathrm{f}$

Murphy-Ullrich, J. E., and Sage, E. H. (2014). Revisiting the matricellular concept. Matrix Biol. 37, 1-14. doi: 10.1016/j.matbio.2014.07.005

Murphy-Ullrich, J. E., and Suto, M. J. (2018). Thrombospondin-1 regulation of latent TGF-beta activation: a therapeutic target for fibrotic disease. Matrix Biol. 68-69, 28-43. doi: 10.1016/j.matbio.2017.12.009

Murphy-Ullrich, J. E., Gurusiddappa, S., Frazier, W. A., and Hook, M. (1993). Heparin-binding peptides from thrombospondins 1 and 2 contain focal adhesion-labilizing activity. J. Biol. Chem. 268, 26784-26789. doi: 10.1016/ s0021-9258(19)74381-6

Nunes, S. S., Outeiro-Bernstein, M. A., Juliano, L., Vardiero, F., Nader, H. B., Woods, A., et al. (2008). Syndecan-4 contributes to endothelial tubulogenesis through interactions with two motifs inside the pro-angiogenic N-terminal domain of thrombospondin-1. J. Cell Physiol. 214, 828-837. doi: 10.1002/jcp. 21281

Oh, E. S., Woods, A., and Couchman, J. R. (1997). Syndecan-4 proteoglycan regulates the distribution and activity of protein kinase C. J. Biol. Chem. 272, 8133-8136. doi: $10.1074 /$ jbc. 272.13 .8133

Olerud, J., Johansson, M., Lawler, J., Welsh, N., and Carlsson, P. O. (2008). Improved vascular engraftment and graft function after inhibition of the angiostatic factor thrombospondin-1 in mouse pancreatic islets. Diabetes. 57, 1870-1877. doi: $10.2337 / \mathrm{db} 07-0724$ 
Orr, A. W., Pedraza, C. E., Pallero, M. A., Elzie, C. A., Goicoechea, S., Strickland, D. K., et al. (2003). Low density lipoprotein receptor-related protein is a calreticulin coreceptor that signals focal adhesion disassembly. J. Cell Biol. 161, 1179-1189. doi: 10.1083/jcb.200302069

Primo, L., Ferrandi, C., Roca, C., Marchio, S., di Blasio, L., Alessio, M., et al. (2005). Identification of CD36 molecular features required for its in vitro angiostatic activity. FASEB J. 19, 1713-1715. doi: 10.1096/fj.05-3697fje

Reinhold, M. I., Lindberg, F. P., Plas, D., Reynolds, S., Peters, M. G., and Brown, E. J. (1995). In vivo expression of alternatively spliced forms of integrin-associated protein (CD47). J. Cell Sci. 108(Pt 11), 3419-3425.

Ren, Y., and Savill, J. (1995). Proinflammatory cytokines potentiate thrombospondin-mediated phagocytosis of neutrophils undergoing apoptosis. J. Immunol. 154, 2366-2374.

Risher, W. C., Kim, N., Koh, S., Choi, J. E., Mitev, P., Spence, E. F., et al. (2018). Thrombospondin receptor alpha2delta-1 promotes synaptogenesis and spinogenesis via postsynaptic Rac1. J. Cell Biol. 217, 3747-3765. doi: 10.1083/ jcb.201802057

Rodriguez-Manzaneque, J. C., Lane, T. F., Ortega, M. A., Hynes, R. O., Lawler, J., and Iruela-Arispe, M. L. (2001). Thrombospondin-1 suppresses spontaneous tumor growth and inhibits activation of matrix metalloproteinase- 9 and mobilization of vascular endothelial growth factor. Proc. Natl. Acad. Sci. U.S.A. 98, 12485-12490. doi: 10.1073/pnas. 171460498

Rohrs, J. A., Sulistio, C. D., and Finley, S. D. (2016). Predictive model of thrombospondin-1 and vascular endothelial growth factor in breast tumor tissue. NPJ Syst. Biol. Appl. 2:16030.

Russell, S., Duquette, M., Liu, J., Drapkin, R., Lawler, J., and Petrik, J. (2015). Combined therapy with thrombospondin-1 type I repeats (3TSR) and chemotherapy induces regression and significantly improves survival in a preclinical model of advanced stage epithelial ovarian cancer. FASEB J. 29, 576-588. doi: 10.1096/fj.14-261636

Russo, T. A., Banuth, A. M. M., Nader, H. B., and Dreyfuss, J. L. (2020). Altered shear stress on endothelial cells leads to remodeling of extracellular matrix and induction of angiogenesis. PLoS One. 15:e0241040. doi: 10.1371/journal.pone. 0241040

Short, S. M., Derrien, A., Narsimhan, R. P., Lawler, J., Ingber, D. E., and Zetter, B. R. (2005). Inhibition of endothelial cell migration by thrombospondin-1 type- 1 repeats is mediated by betal integrins. J. Cell Biol. 168, 643-653. doi: $10.1083 /$ jcb. 200407060

Silverstein, R. L., and Febbraio, M. (2009). CD36, a scavenger receptor involved in immunity, metabolism, angiogenesis, and behavior. Sci. Signal. 2:re3. doi: $10.1126 /$ scisignal.272re3

Simons, M., Gordon, E., and Claesson-Welsh, L. (2016). Mechanisms and regulation of endothelial VEGF receptor signalling. Nat. Rev. Mol. Cell Biol. 17, 611-625. doi: 10.1038/nrm.2016.87

Sipes, J. M., Murphy-Ullrich, J. E., and Roberts, D. D. (2018). Thrombospondins: purification of human platelet thrombospondin-1. Methods Cell Biol. 143, 347-369. doi: 10.1016/bs.mcb.2017.08.021

Soto-Pantoja, D. R., Kaur, S., and Roberts, D. D. (2015). CD47 signaling pathways controlling cellular differentiation and responses to stress. Crit. Rev. Biochem. Mol. Biol. 50, 212-230. doi: 10.3109/10409238.2015.1014024

Soto-Pantoja, D. R., Terabe, M., Ghosh, A., Ridnour, L. A., DeGraff, W. G., Wink, D. A., et al. (2014). CD47 in the tumor microenvironment limits cooperation between antitumor T-cell immunity and radiotherapy. Cancer Res. 74, 67716783. doi: 10.1158/0008-5472.can-14-0037-t

Staniszewska, I., Zaveri, S., Del Valle, L., Oliva, I., Rothman, V. L., Croul, S. E., et al. (2007). Interaction of alpha9betal integrin with thrombospondin-1 promotes angiogenesis. Circ. Res. 100, 1308-1316. doi: 10.1161/01.res.0000266662.98355. 66

Stenina-Adognravi, O., Muppala, S., and Gajeton, J. (2018). Thrombospondins and remodeling of the tumor microenvironment. Vessel Plus 2, 30. doi: 10.20517/ 2574-1209.2018.40

Strickland, D. K., Gonias, S. L., and Argraves, W. S. (2002). Diverse roles for the LDL receptor family. Trends Endocrinol. Metab. 13, 66-74. doi: 10.1016/s10432760(01)00526-4

Sun, J., Hopkins, B. D., Tsujikawa, K., Perruzzi, C., Adini, I., Swerlick, R., et al. (2009). Thrombospondin-1 modulates VEGF-A-mediated Akt signaling and capillary survival in the developing retina. Am. J. Physiol. Heart Circ. Physiol. 296, H1344-H1351.
Sun, X., Mosher, D. F., and Rapraeger, A. (1989). Heparan sulfate-mediated binding of epithelial cell surface proteoglycan to thrombospondin. J. Biol. Chem. 264, 2885-2889. doi: 10.1016/s0021-9258(19)81695-2

Sun, X., Skorstengaard, K., and Mosher, D. F. (1992). Disulfides modulate RGDinhibitable cell adhesive activity of thrombospondin. J. Cell Biol. 118, 693-701. doi: $10.1083 /$ jcb.118.3.693

Tamkun, J. W., DeSimone, D. W., Fonda, D., Patel, R. S., Buck, C., Horwitz, A. F., et al. (1986). Structure of integrin, a glycoprotein involved in the transmembrane linkage between fibronectin and actin. Cell. 46, 271-282. doi: 10.1016/0092-8674(86)90744-0

Tan, K., Duquette, M., Liu, J. H., Dong, Y., Zhang, R., Joachimiak, A., et al. (2002). Crystal structure of the TSP-1 type 1 repeats: a novel layered fold and its biological implication. J. Cell Biol. 159, 373-382.

Tan, K., Duquette, M., Liu, J. H., Shanmugasundaram, K., Joachimiak, A., Gallagher, J. T., et al. (2008). Heparin-induced cis- and trans-dimerization modes of the thrombospondin-1 N-terminal domain. J. Biol. Chem. 283, 39323941. doi: 10.1074/jbc.m705203200

Taraboletti, G., Morbidelli, L., Donnini, S., Parenti, A., Granger, H. J., Giavazzi, R., et al. (2000). The heparin binding $25 \mathrm{kDa}$ fragment of thrombospondin1 promotes angiogenesis and modulates gelatinase and TIMP-2 production in endothelial cells. FASEB J. 14, 1674-1676. doi: 10.1096/fj.99-0931fje

Taraboletti, G., Roberts, D., Liotta, L. A., and Giavazzi, R. (1990). Platelet thrombospondin modulates endothelial cell adhesion, motility, and growth: a potential angiogenesis regulatory factor. J. Cell Biol. 111, 765-772. doi: 10.1083/ jcb.111.2.765

Thorne, R. F., Law, E. G., Elith, C. A., Ralston, K. J., Bates, R. C., and Burns, G. F. (2006). The association between CD36 and Lyn protein tyrosine kinase is mediated by lipid. Biochem. Biophys. Res. Commun. 351, 51-56. doi: 10.1016/ j.bbrc.2006.09.156

Tsai, R. K., Rodriguez, P. L., and Discher, D. E. (2010). Self inhibition of phagocytosis: the affinity of 'marker of self' CD47 for SIRPalpha dictates potency of inhibition but only at low expression levels. Blood Cells Mol. Dis. 45, 67-74. doi: 10.1016/j.bcmd.2010.02.016

van Deventer, S., Arp, A. B., and van Spriel, A. B. (2020). Dynamic plasma membrane organization: a complex symphony. Trends Cell Biol. 31, 119-129. doi: 10.1016/j.tcb.2020.11.004

Vischer, P., Feitsma, K., Schon, P., and Volker, W. (1997). Perlecan is responsible for thrombospondin 1 binding on the cell surface of cultured porcine endothelial cells. Eur. J. Cell Biol. 73, 332-343.

Vischer, P., Volker, W., Schmidt, A., and Sinclair, N. (1988). Association of thrombospondin of endothelial cells with other matrix proteins and cell attachment sites and migration tracks. Eur. J. Cell Biol. 47, 36-46.

Vogel, T., Guo, N. H., Krutzsch, H. C., Blake, D. A., Hartman, J., Mendelovitz, S., et al. (1993). Modulation of endothelial cell proliferation, adhesion, and motility by recombinant heparin-binding domain and synthetic peptides from the type I repeats of thrombospondin. J. Cell Biochem. 53, 74-84. doi: 10.1002/ jcb. 240530109

Vuong, T. T., Reine, T. M., Sudworth, A., Jenssen, T. G., and Kolset, S. O. (2015). Syndecan-4 is a major syndecan in primary human endothelial cells in vitro, modulated by inflammatory stimuli and involved in wound healing. J. Histochem. Cytochem. 63, 280-292. doi: 10.1369/002215541556 8995

Wang, F., Liu, Y. H., Zhang, T., Gao, J., Xu, Y., Xie, G. Y., et al. (2020). Aging-associated changes in CD47 arrangement and interaction with thrombospondin-1 on red blood cells visualized by super-resolution imaging. Aging Cell. 19:e13224.

Weng, T. Y., Huang, S. S., Yen, M. C., Lin, C. C., Chen, Y. L., Lin, C. M., et al. (2014). A novel cancer therapeutic using thrombospondin 1 in dendritic cells. Mol. Ther. 22, 292-302. doi: 10.1038/mt.2013.236

Williams, S. E., Ashcom, J. D., Argraves, W. S., and Strickland, D. K. (1992). A novel mechanism for controlling the activity of alpha 2-macroglobulin receptor/low density lipoprotein receptor-related protein. Multiple regulatory sites for 39kDa receptor-associated protein. J. Biol. Chem. 267, 9035-9040. doi: 10.1016/ s0021-9258(19)50384-2

Winnemoller, M., Schon, P., Vischer, P., and Kresse, H. (1992). Interactions between thrombospondin and the small proteoglycan decorin: interference with cell attachment. Eur. J. Cell Biol. 59, 47-55. 
Woods, A., and Couchman, J. R. (1994). Syndecan 4 heparan sulfate proteoglycan is a selectively enriched and widespread focal adhesion component. Mol. Biol. Cell 5, 183-192. doi: $10.1091 / \mathrm{mbc} .5 .2 .183$

Xie, L., Duncan, M. B., Pahler, J., Sugimoto, H., Martino, M., Lively, J., et al. (2011). Counterbalancing angiogenic regulatory factors control the rate of cancer progression and survival in a stage-specific manner. Proc. Natl. Acad. Sci. U.S.A.. 108, 9939-9944. doi: 10.1073/pnas.1105041108

Yang, Z., Strickland, D. K., and Bornstein, P. (2001). Extracellular matrix metalloproteinase 2 levels are regulated by the low density lipoprotein-related scavenger receptor and thrombospondin 2. J. Biol. Chem. 276, 8403-8408. doi: 10.1074/jbc.m008925200

Yu, H., Tyrrell, D., Cashel, J., Guo, N. H., Vogel, T., Sipes, J. M., et al. (2000). Specificities of heparin-binding sites from the amino-terminus and type 1 repeats of thrombospondin-1. Arch. Biochem. Biophys. 374, 13-23. doi: 10. 1006/abbi.1999.1597
Zhang, X., Kazerounian, S., Duquette, M., Perruzzi, C., Nagy, J. A., Dvorak, H. F., et al. (2009). Thrombospondin-1 modulates vascular endothelial growth factor activity at the receptor level. FASEB J. 23, 3368-3376. doi: 10.1096/fj.09-13 1649

Conflict of Interest: The authors declare that the research was conducted in the absence of any commercial or financial relationships that could be construed as a potential conflict of interest.

Copyright (c) 2021 Morandi, Petrik and Lawler. This is an open-access article distributed under the terms of the Creative Commons Attribution License (CC BY).

The use, distribution or reproduction in other forums is permitted, provided the original author(s) and the copyright owner(s) are credited and that the original publication in this journal is cited, in accordance with accepted academic practice. No use, distribution or reproduction is permitted which does not comply with these terms. 